\title{
Analysis of Multi-Chemical Transmission in the Macro-Scale
}

\section{Journal Article}

\section{Author(s):}

McGuiness, Daniel; Giannoukos, Stamatios; Taylor, Stephen; Marshall, Alan

Publication date:

2020-11

Permanent link:

https://doi.org/10.3929/ethz-b-000453164

\section{Rights / license:}

In Copyright - Non-Commercial Use Permitted

\section{Originally published in:}

IEEE Transactions on Molecular, Biological and Multi-Scale Communications 6(2), https://doi.org/10.1109/TMBMC.2020.3012874 


\title{
Analysis of Multi-Chemical Transmission in the Macro-Scale
}

\author{
Daniel Tunç McGuiness*, Stamatios Giannoukos ${ }^{\dagger}$, Stephen Taylor * and Alan Marshall ${ }^{*}$, Senior Member, IEEE \\ *Department of Electrical Engineering and Electronics University of Liverpool Liverpool UK \\ ${ }^{\dagger}$ ETH Zurich, Department of Chemistry and Applied Biosciences, 8093 Zurich, Switzerland
}

\begin{abstract}
Molecular communications (MC) offers an alternative to established methods (i.e., electromagnetic waves), in areas where the latter might prove ineffective (e.g., underwater or underground) due to the environment's effect on the transmitted signal. In such environments the use of particle (i.e., chemical) based communication may offer a better solution. One of the unique attributes of $\mathrm{MC}$ is its ability to employ chemicals as messengers, and transmitting multiple chemicals concurrently offers the potential to significantly increase the information content of the message. In this work, for the first time, the transmission of multiple chemicals with unique mass-to-charge ratios, was studied experimentally and modeled theoretically. Three modulation methods have been proposed and analyzed based on exploiting the uniqueness of the messenger chemicals. Molecular transmission was achieved using an in-house-built odor generator and detection was accomplished by means of a quadrupole mass analyzer. The noise was analyzed for multiMC and was shown to possess additive white Gaussian noise characteristics with different mean $(\mu)$, but similar variance $\left(\sigma^{2}\right)$ values. It was shown experimentally that multiple chemical transmission is both feasible and advantageous compared to single chemical transmission and the proposed modulation methods exhibit unique advantages that can be used for different scenarios.
\end{abstract}

Index Terms-Macro-scale molecular communication, Closed Boundary, Mass Spectrometer.

\section{INTRODUCTION}

$\mathbf{M}$ OLECULAR communication (MC) is the application of transmitting information by using particles (i.e., molecules, atoms or ions) instead of electromagnetic (EM) waves [1]-[3]. This difference of paradigm allows the implementation of MC in areas where EM might prove impractical or inefficient. For example, in [4] it was shown that currently available wireless sensor networks are insufficient for practical deployment for infrastructure monitoring. Underground communications another area where EM communication experiences substantial losses as it propagates through the environment [5], [6]. However, the applications of MC are not limited to being just an alternative to EM, as it can also be used to study biological species [7]-[9] and imitating them for use in robotic communications [10]-[13].

A primary advantage of $\mathrm{MC}$ is, in itself, not bounded by scale whereas EM has physical limits within which a receiver/transmitter (i.e., antennae) can be designed [14]-[16]. This lower limit of EM has generated interest in MC for use in micro-scale $(\mathrm{nm}-\mu \mathrm{m})$ with application proposals such as: intra-cellular communication [17], drug delivery systems [18] etc.. During these studies, numerous aspects of MC have been analyzed in detail, such as;

- Channel capacity in different mediums [19]-[22],

- Error correction [23]-[25],

- Transmission protocols [26],

- Security [27].

The use of MC in the macro-scale $(\mathrm{cm}-\mathrm{m})$ has seen less interest compared to its micro-scale counterpart. This can be credited to MC's ability to go to smaller scales than is possible with EM [14]-[16] and EM being established as the de-facto standard in long-distance communication. This, however, does not limit its applicability as there is a recent resurgence of experimental analysis of MC in macro-scale. These experiments can range from transmission bits using chemicals [28] all the way to using organisms (i.e., bacteria) [29]. These experiments have shown different aspects of macro-scale: parameter [30], [31], ISI (inter-symbol interference) [32] and MIMO [33] (multiple input multiple output). However, most studies have used only one chemical as a means of transmission. Using a single chemical can create an upper limit of performance for molecular communication, however using multiple chemicals can allow the development of novel modulation methods which increase this limit, thereby increasing the channel capacity. In [34] multiple chemical transmission was conducted however, the transmission was not analyzed, which this study aims to accomplish.

In this study, aspects of multiple chemical transmission are considered, experiments conducted to demonstrate the feasibility of multiple chemical transmission and the results theoretically analyzed. In order to conduct the study, a membrane-inlet mass spectrometer (MIMS) with a quadrupole mass analyzer (QMA) was used as the receiver and an in-house-built odor generator $(\mathrm{OG})$ used as the transmitter. Mass spectrometers (MS) have the ability to analyze and distinguish numerous compounds simultaneously, making it a suitable detector for use in this application [35].

The novel contributions of the paper are as follows;

- Multiple chemical transmission: An experimental along with theoretical study of noise in multiple chemical transmission and multiple chemical transmission was carried out whereby acetone and methanol were transmitted and received concurrently.

- Chemical quadrature amplitude modulation $(\operatorname{ChQAM}(k, m))$ : Influenced by its electromagnetic counterpart, quadrature amplitude modulation, theoretical 


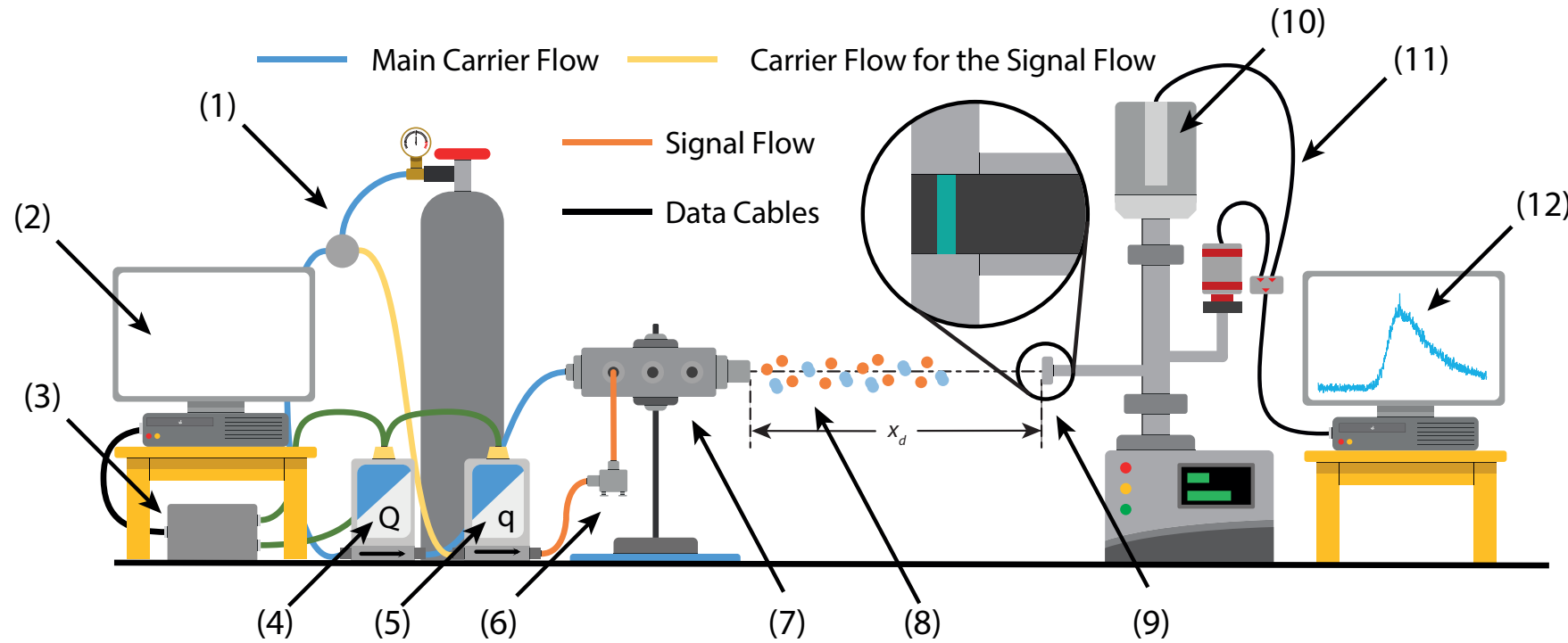

Fig. 1. The diagram of the experimental setup: (1) $\left(\mathrm{N}_{2}\right)$ gas was used as the carrier flow $(Q)$ and was transferred into the MFC, controlling both the carrier flow (blue line) $(Q)$ and the signal flow (yellow line) $(q)$. (2) Modulation information was generated using a computer software. (3) Generated modulation was transmitted into an automation platform where it sends the modulation to the MFC's to create pulses. (4) MFC for the carrier flow. (5) MFC for the signal flow. (6) Evaporation chamber (EC) where the signal chemical was injected into. (7) Mixing chamber where the signal chemicals arrived and initiated the transmission from the transmitter to the detector. (8) Transmission medium. (9) Inlet of the mass spectrometer with a membrane. (10) Mass analyzer where the data was monitored. (11) Controller for the vacuum pump of the mass spectrometer. (12) Controller and the regulator cables for the mass spectrometer.

analysis was carried out on the modulation scheme. This modulation scheme relies on the combination of various chemicals with the same, or different mass concentrations, to transmit information.

- Chemical time-offset keying (ChToK): A second modulation scheme is proposed, based on the relative position of signals. These chemicals are transmitted inside a frame and based on the signals' positions inside the frame, the information can be decoded.

- Chemical ratio keying (ChRK): A third modulation scheme is also proposed, this is designed to exploit the relative ratios between two or more traveling chemicals. This modulation scheme can encode more information into a single pulse of chemicals without increasing the pulse duration.

The structure of the paper is as follows. An introduction to the topic and to the study is given in Section I which also considers the applications and the experimental aspects of macroscale MC. The equipments used in the experimental parts of the study are given in detail in Section II: evaporation chamber (EC) and odor generator (OG) are used for the transmitter part of the setup and the membrane inlet mass spectrometer (MIMS) was used for the detector. Section III presents a description of the equations used throughout the study, based on the solution of the advection-diffusion equation (ADE). The results and the discussions of the study are introduced in Section IV which describes and analyze different aspects of simultaneous molecular transmissions. These include the experimental along with the theoretical transmission of multiple chemical transmission, chemical noise, chemical quadrature amplitude modulation (ChQAM), chemical time offset keying (ChToK) and chemical ratio keying (ChRK). The last section of the study, Section V, is dedicated to conclusions and future work.

\section{EXPERIMENTAL TEST-BED}

To test and study the feasibility of multiple chemical transmission in a macro-scale environment, a bespoke transmitter and a receiver were employed. The signal was generated using an in-house-built odor generator (OG). The detection involved a mass spectrometer (MS) with a quadrupole mass analyzer (QMA). A mass spectrometer is an analytical device that can differentiate a detected chemical by its mass-to-charge $(\mathrm{m} / \mathrm{z})$ ratio. The ability to separate and analyze chemicals in a detected sample makes MS an ideal detector for MC. The experimental setup used for the multi-chemical transmission is shown in Figure 1

\section{A. Transmitter}

The transmitter, shown in Figure 3, consists of three primary parts. The first part consists of mass flow controllers (MFC) which depending on the message going to be transmitted, closes and releases the valves which control the flow of the $N_{2}$ gas. This controlled gas then travels to the evaporation chamber (EC) where the evaporated chemicals in the chamber is carried over to the mixing chamber [34], [36]. For the experiment, volatile organic compounds (VOCs) are used. These types of chemicals have high vapor pressure at room temperature. This property emerges because of the low boiling point of the chemicals.

The second part is the evaporation chamber (EC) where the sample is introduced into the transmitter. The chemicals are evaporated in the chamber and by using the MFC, a flow is generated and carried over to the last part of the transmitter, the mixing chamber. 


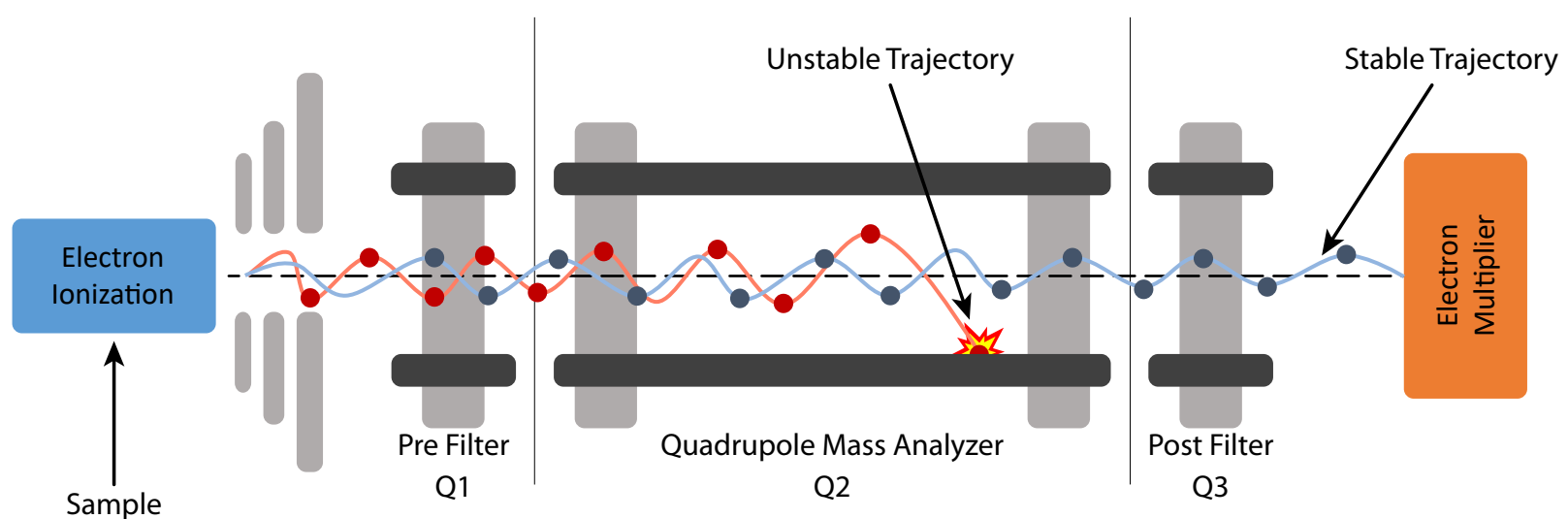

Introduction

Vacuum Environment

Fig. 2. Diagram of the operation of a quadrupole mass analyzer (QMA). The sample was introduced into the system via a suitable interface, which in this case was the membrane inlet (polydimethylsiloxane). The purpose of the membrane inlet is to isolate the vacuum environment from the outside environment where the base pressure of the system was $2.5 \times 10^{-8}$ Torr. For ionization, the sample is bombarded with electrons in the electron ionization (EI) chamber. The ionized particles are then transferred into the quadrupole area via the focus lens (DC). The ions then travel the duration of the path inside the hyperbolic electric field generated via the quadrupole. Only the ions with the correct mass-to-charge ratio values have stable trajectories within the field generated via RF + DC applied to the hyperbolic rods. These ions are safely transported from the initial introduction to the electron multiplier. Ions with unstable trajectories, however, collide with the rods or the walls of the chamber and are neutralized. The stability of each ion passing through the mass analyzer is a function of the DC and RF voltages applied to the rods.

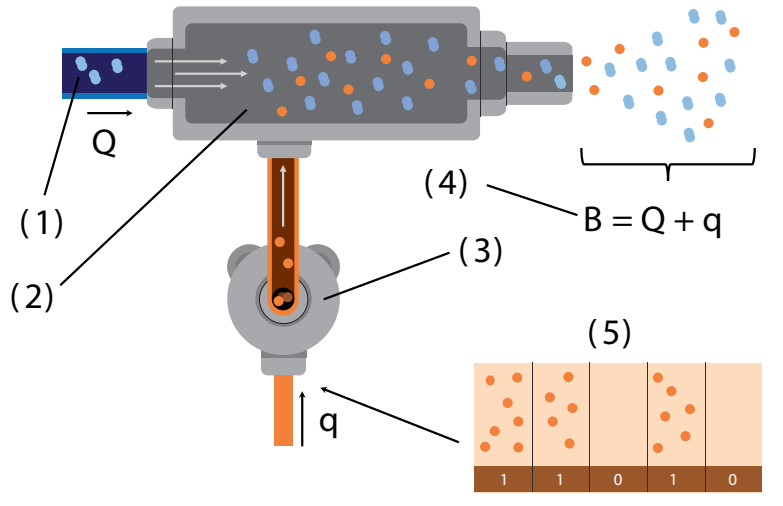

Fig. 3. Diagram of the gas generator (1) Introduction of the carrier gas (Q) into the mixing chamber (2) Mixing chamber where the evaporated chemicals from the chamber and the carrier gas are mixed (3) evaporation chamber (4) Transmitted chemicals that are released from the chamber.

\section{B. Chemical}

In the experiments presented in this study, three types of chemicals were employed as signal chemicals $(q)$. These were chosen as acetone (\% 99.8 purity, CAS Number: 67-64-1), methanol (over \% 99.9 purity), cyclopentane (CAS Number: 287-92-3) and n-hexane (CAS: 110-54-3) which all were supplied by Sigma-Aldrich. Acetone, methanol and n-hexane were introduced to the environment in their liquid phase through the EC and $\mathrm{N}_{2}$ was stored in its gas phase in a pressurized cylinder. A Zero-grade $\mathrm{N}_{2}$ (\% 99.998 purity), supplied by $B O C$ Ltd., was chosen for the carrier gas $(Q)$ to carry both the signal chemical from the transmitter to the detector and transports the signal chemical from the evaporation chamber to the transmitter.
(1)

(2)

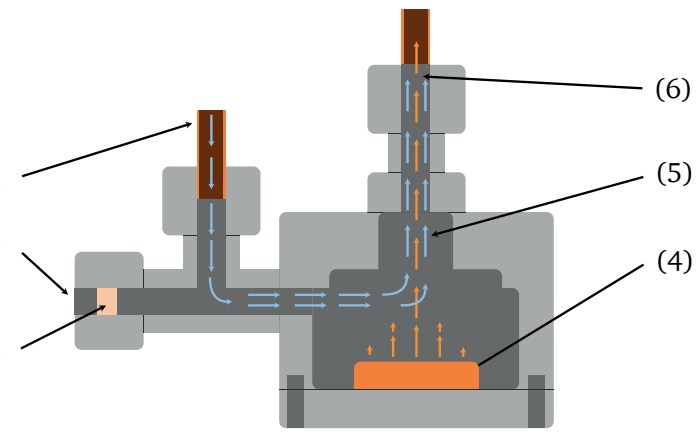

Fig. 4. Diagram of the evaporation chamber: (1) Inlet of the $\mathrm{N}_{2}$ gas into the evaporation chamber (2) Inlet where the sample is introduced (3) thermoresistant septum lets multiple introduction of a sample introduction via a micro syringe (4) An absorptive material holds the liquid sample analyte (5) $\mathrm{N}_{2}$ from the inlet carries the evaporated chemicals from the chamber (6) The cumulated gas it transferred into the mixing chamber via a 0.25 inch Teflon tube.

\section{Detector}

For the experiments, a portable membrane inlet mass spectrometer (MIMS), provided by $Q$ Technologies Ltd., was used as the primary detector for the detection of chemicals. Mass spectrometers play an important role in many fields and applications, such as identifying and monitoring bio-markers in physiological fluids, rapid screening of drug-target binding etc.. As a result of its vast usage in most fields, its versatility and its quantitative and qualitative analysis abilities, MS has become a routinely used technique. They also have the ability to detect numerous chemicals concurrently and with high resolution making it a valuable option for use in molecular communications as a receiver. The applications and the usage of the device are described in detail in literature [37]-[40].

A MIMS is constructed from three primary components: a 
sampling probe which allows the gas sample to penetrate the membrane to allow the MS to analyze, a triple filter quadrupole mass analyzer (QMA), built from an electron ionization source (EI), mass analyzer, vacuum system and a detector. Finally, the inlet of the detector has a non-sterile flat polydimethylsiloxane (PDMS) membrane [34], [37]. The introduction of samples into the evaporation chamber (EC) is via a semi-permeable silicone membrane inlet sampling probe. The membrane is composes of a non-sterile flat PDMS with a thickness of 0.12 $\mathrm{mm}$ and a sampling area of $33.2 \mathrm{~mm}^{2}$. The inner workings of the quadrupole mass analyzer (QMA) can be seen in Figure 2

\section{MOLECUlar Transmission}

A transmission involving particles (i.e., chemicals) can be modeled using the generalized advection-diffusion equation (ADE). Depending on the context, this equation is also known in the literature as convection-diffusion equation.

$$
\frac{\partial c}{\partial t}=\nabla \cdot(D \nabla c)-\nabla \cdot(\mathbf{u} c)+K
$$

where $c$ is the concentration of the mass $(\mathrm{kg} / \mathrm{m}), t$ is the duration of mass transfer process (s), $D$ is the coefficient of diffusivity $\left(\mathrm{cm}^{2} / \mathrm{s}\right), \mathbf{u}$ is the velocity vector $(\mathrm{cm} / \mathrm{s})$ and $K$ is the sink and/or the source.

In this model it is assumed the environment possesses neither a sink nor a source $(K=0)$ and the diffusion stays constant $(\partial D / \partial t=0)$ during the propagation process. The solution for this equation can be derived based on the following initial boundary conditions, known as the "thin-film solution" in the literature [41]:

$$
\begin{array}{r}
c\left(|x|>0, t_{0}\right)=0, \\
c\left(x=0, t_{0}\right)=M_{0} \delta(x), \\
c(|x| \rightarrow \infty, t)=0 .
\end{array}
$$

where $M_{0}$ is the initial mass injected into the environment $(\mathrm{kg}), x$ is the Cartesian propagation dimension, $t_{0}$ is the initial time (s) and $\delta(x)$ is the dimensional Dirac delta function. Based on these conditions to the partial differential equation, the solution with an unbounded domain, can be expressed as:

$$
c(x, t)=\frac{M_{0}}{\sqrt{(4 \pi D t)}} \exp \left(-\frac{\left(x-u_{x} t\right)^{2}}{4 D t}\right),
$$

where $u_{x}$ is the vectorial elements of $\mathbf{u}$ and $D_{x}$ is the vectorial elements of $D$. The diffusion coefficient $(D)$ can be estimated as follows;

$$
D=\frac{2}{3} \sqrt{\frac{k_{\mathrm{B}}^{3} T^{3}}{\pi^{3}}} \sqrt{\frac{1}{2 m_{\mathrm{A}}}+\frac{1}{2 m_{\mathrm{B}}}} \frac{4}{P\left(d_{\mathrm{A}}+d_{\mathrm{B}}\right)^{2}},
$$

where $k_{B}$ is the Boltzmann constant $\left(k_{B}=1.380609 \times\right.$ $\left.10^{-23} \mathrm{~J} \times \mathrm{K}^{-1}\right), P$ is pressure, $T$ is temperature, $m_{A}, m_{B}$ and $d_{A}, d_{B}$ are the molecular masses and diameters of chemical $\mathrm{A}$ and $\mathrm{B}$ respectively. The Eq. (3) quantifies the concentration value of the sample in a given time $(t)$ and space $(x)$. The mass in a given transmission $(\theta)$ can be calculated by integrating the concentration function in the desired volume:

$$
\theta=\int C d x
$$

As mentioned, the system has no sink/source $(K=0)$ therefore, the chemicals used in the transmission can either be in transmission $\left(\theta_{T}\right)$ or have been absorbed by the detector $\left(\theta_{A}\right)$. Therefore, both the aforementioned mass values must add up to the initial introduction of mass in the beginning of the transmission.

$$
M=\theta_{T}+\theta_{A}
$$

The absorbed mass $\left(\theta_{A}\right)$ can be calculated simply by subtracting from the initial mass $(M)$.

$$
\theta_{A}=M-\theta_{T}
$$

By integrating the concentration function with respect to space, the particles present in the environment can be calculated. To calculate the chemicals absorbed by the detector, the integration function is subtracted from the injected mass [30], [31], [42].

$$
\theta(x, t)=M-\int_{-x_{\epsilon}}^{+x_{d}} C(x, t) d x,
$$

where $x_{d}$ is the distance from the detector to the origin point $(x=0)(\mathrm{m})$ and $x_{\epsilon}$ is the distance particles travel against the flow (m). The solution to the integration given in Eq. (8) for transmission with no boundaries is given in Eq. (9).

$$
\begin{aligned}
& \theta_{1}\left(x_{d}, x_{\epsilon}, t\right)=M \\
& -\frac{M}{2}\left[\operatorname{erf}\left(\frac{x_{d}-u_{x} t}{\sqrt{4 D_{x} t}}\right)+\operatorname{erf}\left(\frac{x_{\epsilon}+u_{x} t}{\sqrt{4 D_{x} t}}\right)\right] .
\end{aligned}
$$

where $\operatorname{erf}(x)=2 / \sqrt{\pi} \int_{0}^{x} e^{-t^{2}} d t$. The chemicals absorbed by the detector $\left(\theta_{1}\right)$ in a given period of $T$ is given below:

$$
M_{R}=\theta_{1}\left(x_{d}, t=T\right)-\theta_{1}\left(x_{d}, t=0\right) .
$$

Therefore, the removal of chemicals from the detector $\left(\theta_{0}\right)$ to the outside environment can be expressed by the following expression.

$$
\begin{aligned}
& \theta_{1}\left(x_{d}, x_{\epsilon}, t\right)= \\
& \frac{M_{R}}{2}\left[\operatorname{erf}\left(\frac{x_{d}-u_{x} t}{\sqrt{4 D_{x} t}}\right)+\operatorname{erf}\left(\frac{x_{\epsilon}+u_{x} t}{\sqrt{4 D_{x} t}}\right)\right] .
\end{aligned}
$$

As can be seen from Eq. (9) and Eq. (11), the mass parameter is different in each equation: former being the mass injected into the environment $(M)$ and the latter is the mass absorbed by the detector $\left(M_{R}\right)$. The application and the process of introduction/removal of particles in $1 \mathrm{D}$ can be seen in detail in [30], [31]. 
TABLE I

EXPERIMENTAL PARAMETERS FOR MULTI-ION TRANSMISSION

\begin{tabular}{rccc}
\hline Parameter & Symbol & Value & Unit \\
\hline Tracked acetone, methanol ion & $m / z$ & 43,31 & $\mathrm{Da}$ \\
Bit 1, 0 signal flow & $q_{1}, q_{2}$ & 8,0 & $\mathrm{ml} / \mathrm{min}$ \\
Carrier flow & $Q$ & 750 & $\mathrm{ml} / \mathrm{min}$ \\
Carrier flow pressure & $P_{F}$ & 1 & $\mathrm{bar}$ \\
Symbol duration & $T$ & 60 & $\mathrm{~s}$ \\
Vacuum pump pressure & $P_{V}$ & $3 \times 10^{-6}$ & torr \\
Temperature & $T_{E}$ & $299.35 \pm 1.5$ & $\mathrm{~K}$ \\
Pressure & $P_{E}$ & $0.973 \pm 0.001$ & $\mathrm{bar}$ \\
Transmission distance & $x_{d}$ & $2.5 \pm 0.1$ & $\mathrm{~cm}$ \\
Diffusion of acetone, methanol in air & $D_{A}, D_{M}$ & $0.124,0.15$ & $\mathrm{~cm}^{2} / \mathrm{s}$ \\
Acetone detection delay 34$]$ & $t_{d}$ & 15 & $\mathrm{~s}$ \\
\hline
\end{tabular}

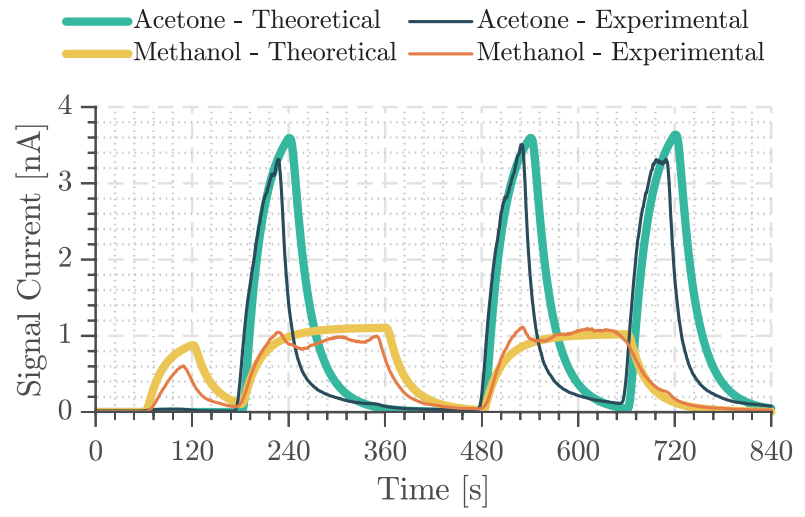

Fig. 5. Experimental along with theoretical results of multi-chemical transmission.

\section{RESULTS}

\section{A. Multi Chemical Transmission}

In this part of the study, an experiment was conducted to test the feasibility of sending two chemicals simultaneously. To detect and analyze multiple chemicals concurrently, a mass spectrometer was used, where the operating principle was shown in Figure 2. The signal chemicals $(q)$ were chosen as acetone with a characteristic mass spectral peak (tracked ion) of $43 \mathrm{Da}$ and methanol with tracked ion of $31 \mathrm{Da}$. The experimental and environmental parameters can be seen in Table II

The experimental result of the transmission along with the theoretical comparison can be observed in Figure 5. As can be seen two chemicals (acetone \& methanol) were transmitted simultaneously. However, the difference in the amplitude from the captured particle of two chemical species needs a mention. This can be caused by numerous effects, such as the particles' interaction with the detector inlet (i.e., the PDMS membrane), the presence of another message particle's at the same $\mathrm{m} / \mathrm{z}$ (an isotopologue), and the ionization efficiency of the samples inside the detector. In this transmission the acetone produced a higher signal current than the methanol. However, the retrieved signal of methanol (31 Da) experienced a considerable amount of distortion compared to acetone (43 Da). This effect is possibly due to the diffusion of methanol through the membrane.
As seen in the molecular noise in Figure 6 the $43 \mathrm{Da}$ noise variance is not considerably higher but it is the chemical's presence (i.e., methanol) and its interaction with the PDMS membrane that causes the distortion to occur.

The mathematical model presented in Section III and its comparison to experimental data can be seen in Figure 5 As can be seen, the model shows agreement with the experimental data, with noticeable variations from the theory. This unwanted behavior can be caused by the chemical noises present in the environment and the chemicals' interaction between each other. To quantify the agreement with experimental data to the theoretical model the Pearson correlation is used:

$$
\rho_{E, T}=\frac{\operatorname{cov}(E, T)}{\sigma_{E} \sigma_{T}}
$$

where $E$ is the experimental data and $T$ is the theoretical data. Based on Eq. (12), acetone produced a correlation coefficient of $\rho_{A}=0.86$ and methanol produced a correlation coefficient of $\rho_{M}=0.95$ showing the model's validity in analyzing and predicting multiple chemical transmission. A final comment needs to be made concerning the initial pulsation of the methanol signal. This "transient" behavior can be caused by the complex interactions of the membrane and the chemicals. After the initial interaction the signal becomes reliably predictable by the model developed in Section IIII To understand the behavior of these signals, the noise needs to be analyzed which is the focus of the following subsection.

\section{B. Multi Chemical Noise}

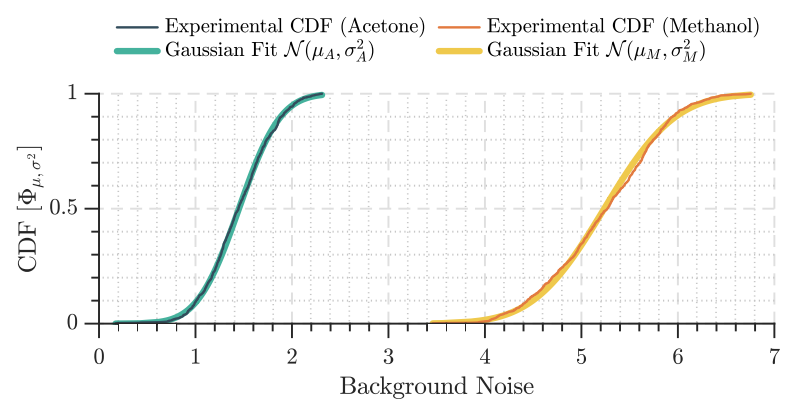

Fig. 6. Experimental along with theoretical fitting of multi-chemical noise.

One of the important properties in communications is the disturbances in the environment which influences the transmission. The noise is therefore needs to be analyzed and identified. The noise present in this form of MC can be caused by numerous effects. These include particles present in the environment, minute pressure differences in the inlet of the detector, and the temperature and pressure of the environment etc.. A study in [30] experimentally shows the noise of a single chemical in the environment effecting the transmission is additive white Gaussian noise (AWGN).

In this experiment, the presence of multiple chemicals noise is conducted to analyze the noise in a multi channel transmission. The experimental parameters can be seen in Table [II The results can be seen in Figure 6 
TABLE II

EXPerimental Parameters of MUlti-CHEMiCAl Noise

\begin{tabular}{rccc}
\hline Parameter & Symbol & Value & Unit \\
\hline Tracked acetone, methanol ion & $m / z$ & 43,31 & $\mathrm{Da}$ \\
Carrier flow pressure & $P_{F}$ & 1 & bar \\
Vacuum pump pressure & $P_{V}$ & $2.85 \times 10^{-6}$ & torr \\
Environmental temperature & $T_{E}$ & 291.85 & $\mathrm{~K}$ \\
Environmental pressure & $P_{E}$ & 1.005 & $\mathrm{bar}$ \\
Diffusion of acetone, methanol in air & $D_{A}, D_{M}$ & $0.124,0.15$ & $\mathrm{~cm}^{2} / \mathrm{s}$ \\
\hline
\end{tabular}

As can be seen the results show a strong relation to Gaussian distribution. To quantify the goodness-of-fit, KolmogorovSmirnov test is used [43]. The equation of this test is given below.

$$
D_{n}=\sup _{x}\left|F_{n}(x)-F(x)\right|
$$
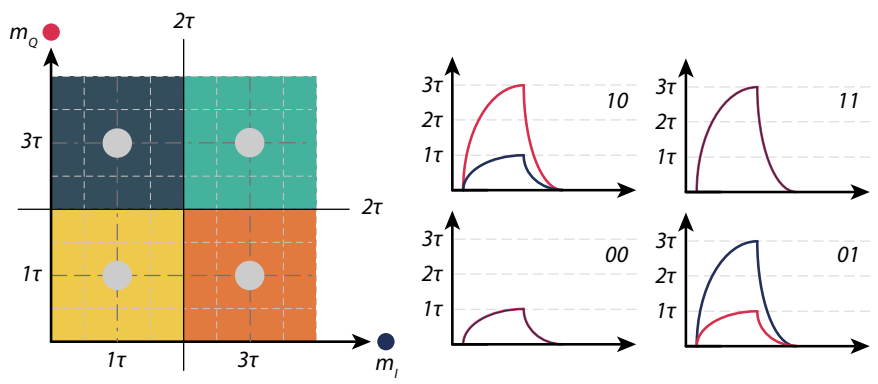

Fig. 7. A constellation diagram for use in molecular quadrature amplitude modulation $\operatorname{ChQAM}(k, 2)$.

Based on the test, the distance for acetone is $D_{n}=0.0267$ and for methanol is $D_{n}=0.0448$ show the noise data is drawn from a Gaussian distribution with a significance level of $\alpha=0.05$. From these results it can be inferred that each chemical possesses a different noise mean $\left(\mu_{N}\right)$ and variance $\left(\sigma_{\mathrm{N}}^{2}\right)$. In addition, from Figure 5 , different peak values for acetone and methanol can be observed. This behavior shows that each chemical has a different signal-to-noise ratio (SNR), which can make the process of choosing the best chemical for transmission for an environment an optimization problem in itself. Based on the transmission feasibility and noise properties, different modulation methods can therefore be developed that exploit the multiple chemical transmission. The first one developed is to use amplitude and chemical type concurrently, which is the focus of the next subsection.

\section{Chemical Quadrature Amplitude Modulation (ChQAM)}

By using more than a single chemical to transmit information, the amount of bits encoded in a given symbol can be increased, which in turn increases the channel capacity $(C)$ of the transmission.

In this section, a molecular analogy of quadrature amplitude modulation (QAM) is developed, whereby coding a bit value into the amplitude value of more than one chemical, increases the number of bits in a symbol.
The amount of mappable elements onto a molecular amplitude can be expressed by the cardinality (i.e., number of elements in a set) of the input set with following equation:

$$
\operatorname{card}(\operatorname{ChQAM}(k, m))=k^{m},
$$

where $k$ is the number of modulated levels on the chemicals and $m$ is the number of distinguishable chemicals used in the transmission of information. Based on this, the channel capacity of an $n$ block transmission with memory effects can be written using the following generalized equation [44]:

$$
C \triangleq \lim _{x \rightarrow \infty} \sup \frac{1}{n} I\left(X^{n} ; Y^{n}\right) .
$$

In the above equation $I\left(X^{n} ; Y^{n}\right)$ represents the mutual information between the input alphabet $X$ and the output $Y$ with the following identity [45]:

$$
I\left(X^{n} ; Y^{n}\right)=H\left(Y^{n}\right)-H\left(Y^{n} \mid X^{n}\right),
$$

where $H\left(Y^{n}\right)$ is the Shannon entropy of the probability vector $Y$ and $H\left(Y^{n} \mid X^{n}\right)$ is the conditional entropy of $Y$ given $X$.

$$
\begin{aligned}
\mathrm{H}(X) & =-\sum_{i=1}^{n} p\left(x_{i}\right) \log _{2} p\left(x_{i}\right), \\
\mathrm{H}(Y \mid X) & =-\sum_{x \in \mathcal{X}} p(x) \sum_{y \in \mathcal{Y}} p(y \mid x) \log _{2} p(y \mid x) .
\end{aligned}
$$

In macro-scale molecular communication the effects of ISI on the channel behavior needs a brief discussion. Molecular communication, as stated, uses particles to instigate transmission and as the transmission evolves the leftover chemicals in the environment and/or the detector may pose a hindrance on the achievable information rate. This can cause incorrect decoding at a different rate (i.e., $p(0 \mid 1) \neq p(1 \mid 0)$ ). Therefore, every value in the probability matrix needs to be calculated individually as the ISI may effect the values unevenly making the behavior of the channel asymmetric [46].

As indicated, a unique attribute of molecular communication is the ability to increase the amount of carrier signals in a given channel. For example, in the experimental analysis shown in Figure 5, transmitting multiple chemicals at the same time is possible. This, however, doesn't limit the number of concurrently usable chemicals to two as more chemicals can be used simultaneously. These chemicals have the possibility of transmitting information with negligible interference when chosen carefully (i.e., when chemicals have no alignment in their mass spectrum).

Based on the experimental results shown in Figures 5 and 6. a theoretical analysis was carried out on multiple chemical transmission for ChQAM. In this analysis, two chemicals $\left(m_{I}, m_{Q}\right)$ were chosen for the scenario. It is assumed the messenger chemicals have no alignment in their mass spectrum which can cause interference. The number of modulated levels ( $k$ ) will be $k=\{2,4,8\}$. The constellation diagram used in the simulations can be seen in Figure 7 . To theoretically define 


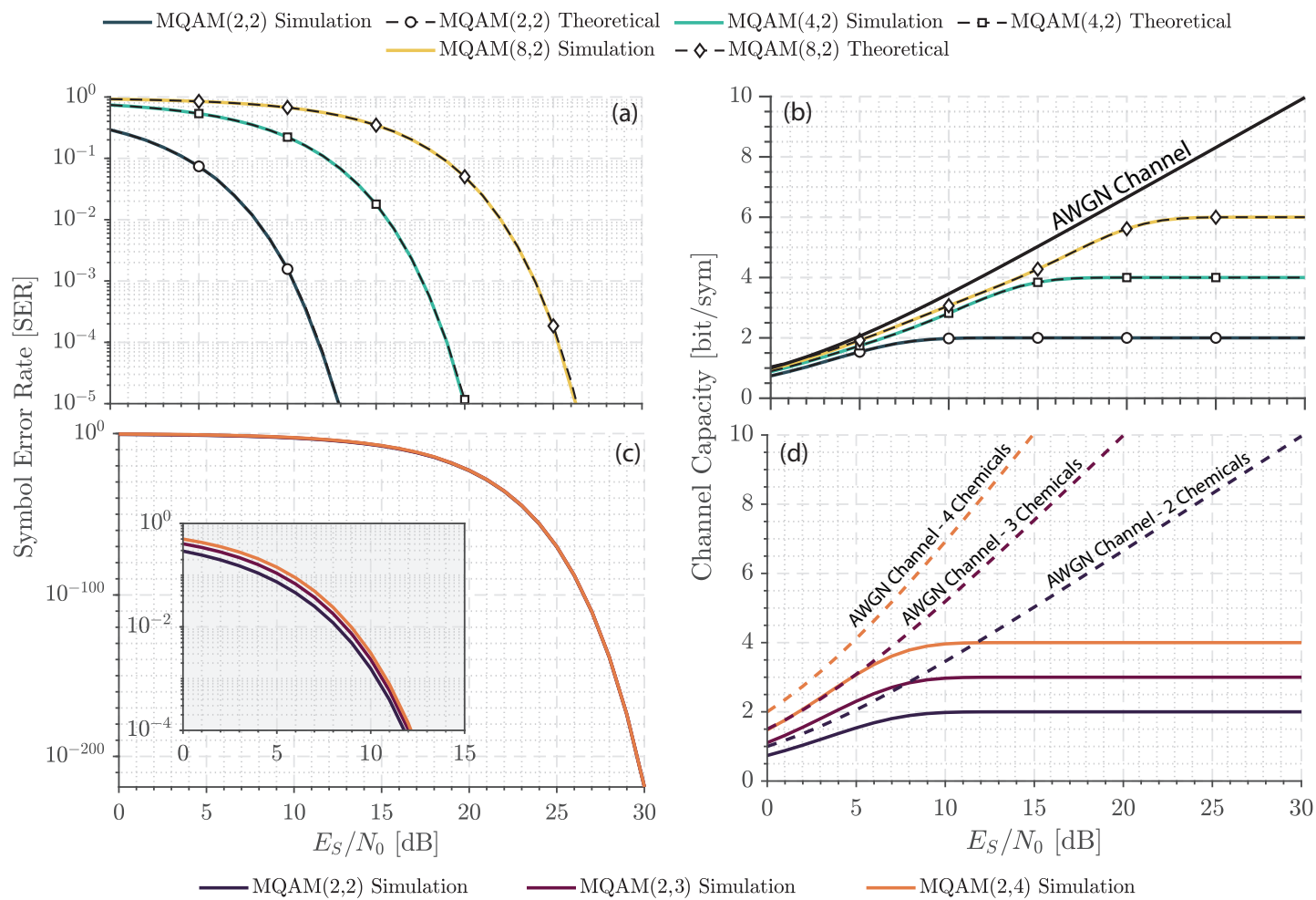

Fig. 8. Theoretical and simulation comparison of (a) symbol error rate (SER) and (b) mutual information (MI) of ChQAM of and ideal scenario of ChQAM $(k, 2)$ where $k=\{2,4,8\}$. Theoretical comparison of ChQAM $(2, m)$ where $m=\{2,3,4\}$ for (c) SER and (d) MI.

the SER and to validate the proposed model, multivariate normal distribution is employed. The probability density function (PDF) of of the distribution is given as;

$$
\begin{aligned}
f(\mathbf{x} ; \mu, \boldsymbol{\Sigma})= & \frac{1}{\sqrt{(2 \pi)^{d}|\mathbf{\Sigma}|}} \\
& \times \exp \left(-\frac{1}{2}(\mathbf{x}-\boldsymbol{\mu})^{\mathrm{T}} \boldsymbol{\Sigma}^{-1}(\mathbf{x}-\boldsymbol{\mu})\right),
\end{aligned}
$$

where $d$ is the number of dimensions, $\mu$ is the expected value of $\mathbf{x}$ (i.e., $\mu=\mathbb{E}[\mathbf{x}]$ ) and $\boldsymbol{\Sigma}$ is the covariance matrix with the following identity,

$$
\boldsymbol{\Sigma}=\operatorname{cov}\left[\mathbf{x}_{i}, \mathbf{x}_{j}\right]=\mathbb{E}\left[\left(\mathbf{x}_{i}-\mathbb{E}\left[\mathbf{x}_{i}\right]\right)\left(\mathbf{x}_{j}-\mathbb{E}\left[\mathbf{x}_{j}\right]\right)\right],
$$

and $|\boldsymbol{\Sigma}|$ is the determinant of $\boldsymbol{\Sigma}$. To calculate the probability occurring during transmission, the conditional probability of the distribution is applied [47].

$$
\mathbf{P}\left[\begin{array}{l}
a_{x} \leq x \leq b_{x} \\
a_{y} \leq y \leq b_{y}
\end{array}\right]=\int_{a_{x}}^{b_{x}} \int_{a_{y}}^{b_{y}} f_{X, Y}(x, y) d y d x
$$

Based on these descriptions the SER and the MI of ChQAM can be seen collectively in Figure 8. In Figures $8 \mathrm{a}$ and $8 \mathrm{~b}$ the theoretical values of SER and MI generated from the aforementioned equation is compared to the simulation of an ideal transmission for ChQAM $(k, 2)$. In this scenario, an ideal transmission is the transmission with minimum distance with maximum advective flow (i.e., square waves). As mentioned before, this modulation method is not bounded by the number of usable chemicals and this is shown in $8 \mathrm{c}$ and $8 \mathrm{~d}$ with $m=$ $\{2,3,4\}$.
TABLE III

Theoretical Parameters of Chemical Quadrature Amplitude MODULATION CHQAM

\begin{tabular}{rccc}
\hline Theoretical Parameter & Symbol & Value & Unit \\
\hline Injected mass & $M_{0}$ & 1 & $\mathrm{ng}$ \\
Transmission distance & $x_{d}$ & 2.5 & $\mathrm{~cm}$ \\
Advective flow in $x$-axis & $u_{x}$ & 0.1 & $\mathrm{~cm} / \mathrm{s}$ \\
Diffusivity & $D$ & 0.1 & $\mathrm{~cm}^{2} / \mathrm{s}$ \\
Symbol duration & $T_{S}$ & 20 & $\mathrm{~s}$ \\
\hline
\end{tabular}

As can be seen, the maximum information (compared to Shannon's law [48]) sees a considerable increase with each chemical species introduced, however the errors produced from the constellation experiences an increase as well. This is due to the increase of symbols which can interfere with their neighboring symbol values. In a constellation with $s^{2}$ number of symbols, the number of possible wrong decoding is $s^{2}-s$. The theoretical upper bound symbol-error rate (SER) for the ChQAM $(k, 2)$ with white Gaussian noise present in the transmission can be given as:

$$
\begin{aligned}
& p(e \mid \mathrm{ChQAM}) \simeq 2\left(1-\frac{1}{k}\right) \operatorname{erfc}\left(\sqrt{\frac{3}{2\left(k^{2}-1\right)} \frac{E_{s}}{N_{0}}}\right) \\
&-\left(1-\frac{2}{k}+\frac{1}{k^{2}}\right) \operatorname{erfc}^{2}\left(\sqrt{\frac{3}{2\left(k^{2}-1\right)} \frac{E_{s}}{N_{0}}}\right)
\end{aligned}
$$




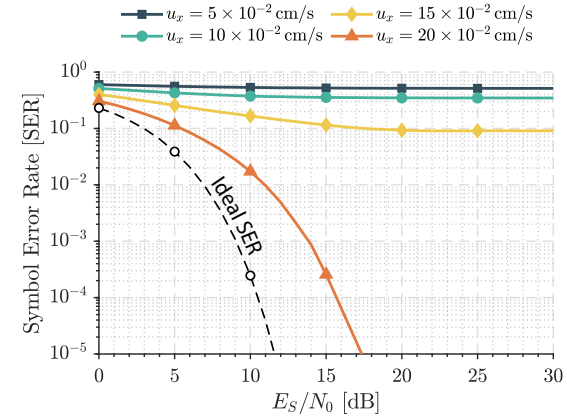

(a)

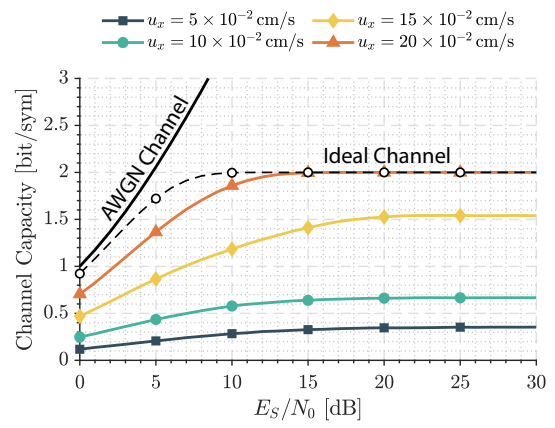

(d)

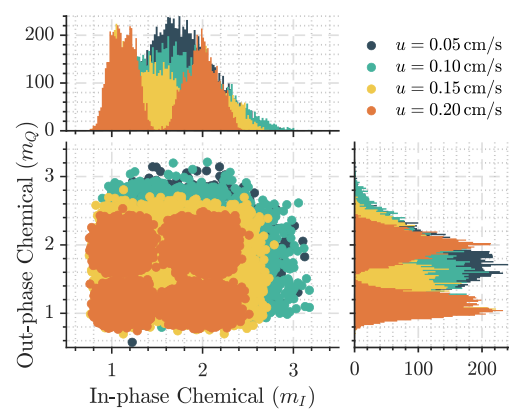

(g)

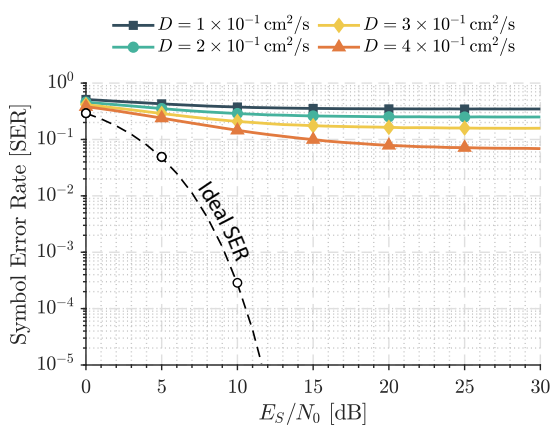

(b)

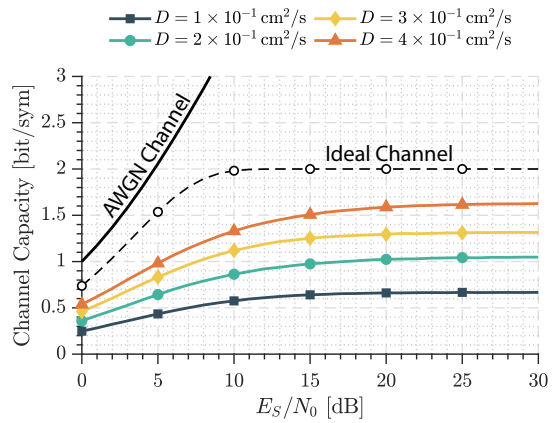

(e)

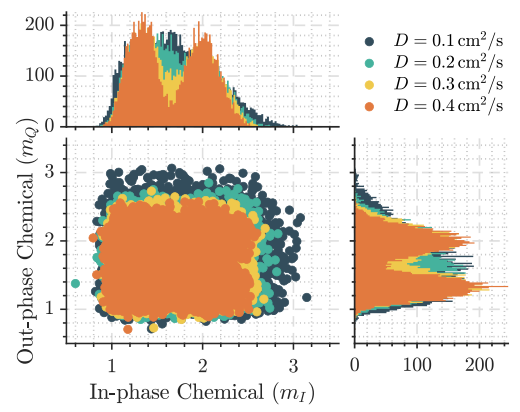

(h)

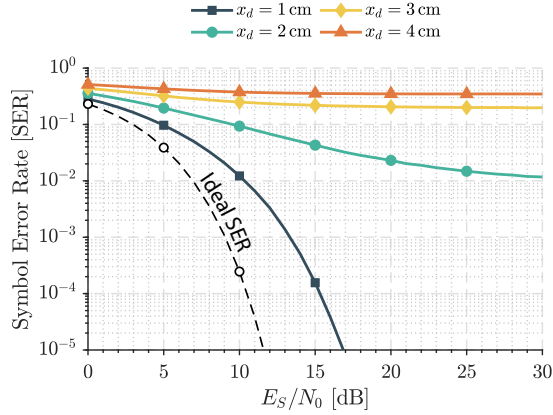

(c)

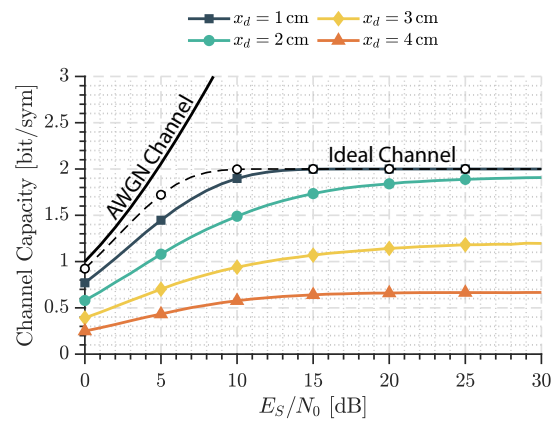

(f)

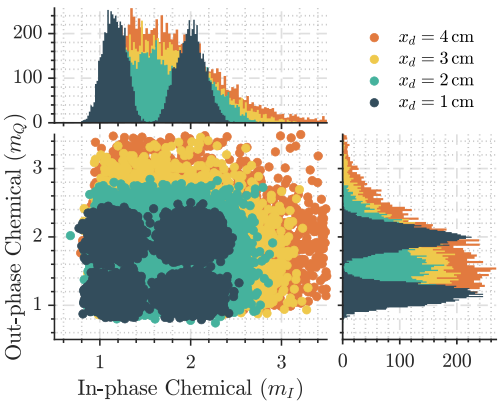

(i)

Fig. 9. SER simulation results for (a) Advective flow (b) Diffusion (c) Transmission distance. MI simulation results for (d) Advective flow (e) Diffusion (f) Transmission distance. Constellation diagram simulation results for (g) Advective flow (h) Diffusion (i) Transmission distance.

where $E_{s} / N_{0}$ is the energy per symbol $(W)$ and $\operatorname{erfc}(x)=$ $1-\operatorname{erf}(x)$. If more than two chemicals are used and modulated using ChQAM, then Eq. 20 can be used to calculate the error probabilities. Though there are no closed form solutions to Eq. [20), it can be still be calculated numerically [47].

The following subsection will focus on the effect of the three parameters (diffusion, advective flow and transmission distance) on the MI and SER of the ChQAM. The values used in the theoretical study can be seen in Table III]

1) Advective Flow: Advective flow $\left(u_{x}\right)$ plays an important role in macro-scale MC. As it can be seen, the increase of flow has a profound effect on the position of the detected mass values $\left(m_{I}, m_{Q}\right)$. This is caused by the $u_{x} t$ part of the equation. As velocity is increased it needs less time to catch up with the distance parameter $\left(x_{d}\right)$ which increases the likelihood of more particles to be correctly decoded. Lower velocity values have shown that the detected bit values are scattered away from the ideal value. The distribution of masses with different advective flow can be seen in Figure 9g. The theoretical analysis of $\operatorname{ChQAM}(2,2)$ analysis for different advective flows can be seen in Figure 9a for SER and Figure 9d for MI. As mentioned, advective flow plays a positive role in increasing the MI and decreasing the SER encountered during transmission.

2) Coefficient of Diffusivity: Diffusivity plays a lesser role on the transmission compared to the effects of advective flow or transmission distance as a whole which can be observed in Figure $9 \mathrm{~h}$. While the effect is necessary for transmission without external energy, when an active transport is present, the effect of diffusivity does not play a primary role on the clarity of the detected bits. This is likely due to the 


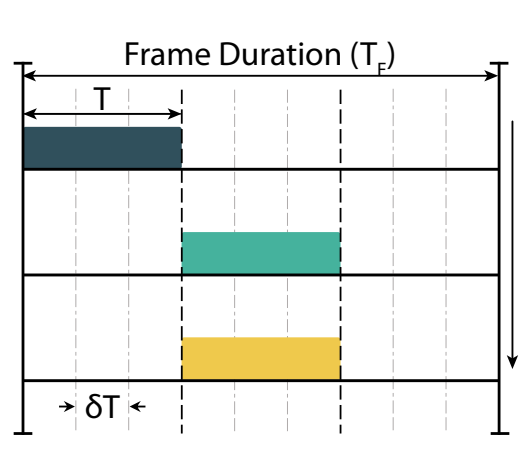

(a)

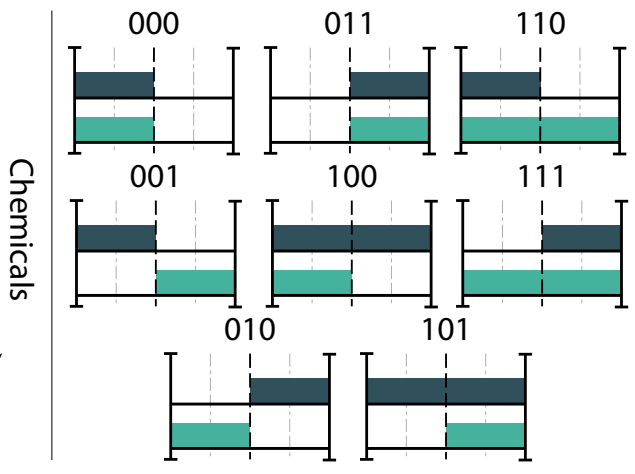

(b)



(c)

Fig. 10. Chemical Time offset Keying (ChToK): (a) The modulation diagram of ChToK where the symbols are defined by their relative positions to each other. (b) The possible combinations present in a $\operatorname{ChToK}(2,2,1)$ (c) A possible application of the proposed modulation is to use one of the chemicals as clock to maintain a synchronization between the transmitter and the receiver.

dominance of the advective flow over the diffusivity of the transmitted chemicals. This behavior can be explained using Péclet number (product of the Reynolds number and the Schmidt number). The Péclet number (Pe) is a dimensionless number used to measure the relative importance of advection versus diffusion, where a large number indicates an advectively dominated distribution, and a small number indicates a diffuse flow [31], [49].

$$
\mathrm{Pe}=x_{d} \frac{u_{x}}{D}
$$

This effect can also be observed in SER and MI in Figure $9 \mathrm{~b}$ and Figure $9 \mathrm{e}$ respectively. As can be seen from both these graphs the effect of increasing the diffusivity coefficient does not increase the separation of the symbols as significant as the advective flow. This effect is caused by the behavior of the signal with respect to advective flow and diffusion. When advective flow is present, the chemical are transmitted within the allocated time through the flow (i.e., $\mu=u_{x} t$ ). However, diffusion takes the role of the variance of the signal (i.e., $\sigma^{2}=$ $4 D t$ ), thereby effecting only the curvature of the signal. If the transmission action is achieved by the use of a flow, the effect of the diffusion becomes comparably less.

3) Transmission Distance: The effect of the transmission distance on detection plays a detrimental role compared to the advective flow. As can be seen when transmission distance $\left(x_{d}\right)$ is increased,the divergence of the received bits from the ideal value also increases, causing wrong decoding of symbols. This can be seen in Figure 9i In addition, as distance is decreased, the concentration of received bits approach closer to the ideal value. This behaviour is caused by the shifting of the mean $\left(x_{d}-u_{x} t\right)$. As the distance increases, so does $u_{x} t$ to keep up the performance. This can be achieved either by increasing the symbol period $(T)$ or the advective flow $\left(u_{x}\right)$. This effect can also be observed in SER and MI in Figure 9c and Figure 9f] respectively.

\section{Chemical Time Offset Keying (ChToK)}

An alternative way of modulating the signal is to include relative relations between each transmitted signal. As it is in EM communication, an analogue of phase shift keying (PSK)
TABLE IV

Parameters for Chemical Time OfFSet Keying (ChToK) Study

\begin{tabular}{rccc}
\hline Experimental Parameter & Symbol & Value & Unit \\
\hline Tracked signal flow ion & $m / z$ & $31^{1}, 43^{2}$ & Da \\
Signal flow & $q$ & 8 & $\mathrm{ml} / \mathrm{min}$ \\
Carrier flow & $Q$ & 750 & $\mathrm{ml} / \mathrm{min}$ \\
Acetone detection delay [34] & $t_{d}$ & 20 & $\mathrm{~s}$ \\
Transmission distance & $x_{d}$ & $2.5 \pm 0.1$ & $\mathrm{~cm}$ \\
Environment pressure & $P_{E}$ & $1 \pm 0.003$ & $\mathrm{bar}$ \\
Carrier flow pressure & $P_{F}$ & 1 & $\mathrm{bar}$ \\
Vacuum pump pressure & $P_{V}$ & $1.95 \times 10^{-6}$ & $\mathrm{torr}$ \\
Environment temperature & $T_{E}$ & $297.35 \pm 1.5$ & $\mathrm{~K}$ \\
Diffusivity in air ${ }^{3}$ & $D$ & $0.15^{1}, 0.124^{2}$ & $\mathrm{~cm}{ }^{2} / \mathrm{s}$ \\
\hline Theoretical Parameter & Symbol & Value & $\mathrm{Unit}$ \\
\hline Advective flow in $x$-axis & $u_{x}$ & 0.12 & $\mathrm{~cm} / \mathrm{s}$ \\
Transmission distance & $x_{d}$ & 2.5 & $\mathrm{~cm}$ \\
Diffusivity & $D$ & $0.15^{1}, 0.124^{2}$ & $\mathrm{~cm}{ }^{2} / \mathrm{s}$ \\
Noise mean \& variance & $\mu_{N}, \sigma_{N}^{2}$ & $1.21,0.096$ & $\mathrm{pA}, \mathrm{W} / \mathrm{ion}$ \\
\hline
\end{tabular}

${ }^{1}$ methanol ${ }^{2}$ acetone

is experimented on molecular communication in this study. The diagram of this modulation can be seen in Figure 10 where $T$ is the period of the pulse of a chemical (s), $\delta T$ is the time frame which the chemical can be shifted (s) and $T_{F}$ is the duration of the frame (s). Therefore the cardinality of the modulation can be represented as:

$$
\operatorname{card}\left(\operatorname{ChToK}\left(\frac{T_{F}}{\delta T}, m\right)\right)=\left(\frac{T_{F}}{\delta T}\right)^{m}
$$

If number of modulation levels $(k)$ are also implemented, the equation can be rewritten as:

$$
\operatorname{card}\left(\operatorname{ChToK}\left(k, \frac{T_{F}}{\delta T}, m\right)\right)=k^{m}\left(\frac{T_{F}}{\delta T}\right)^{m}
$$




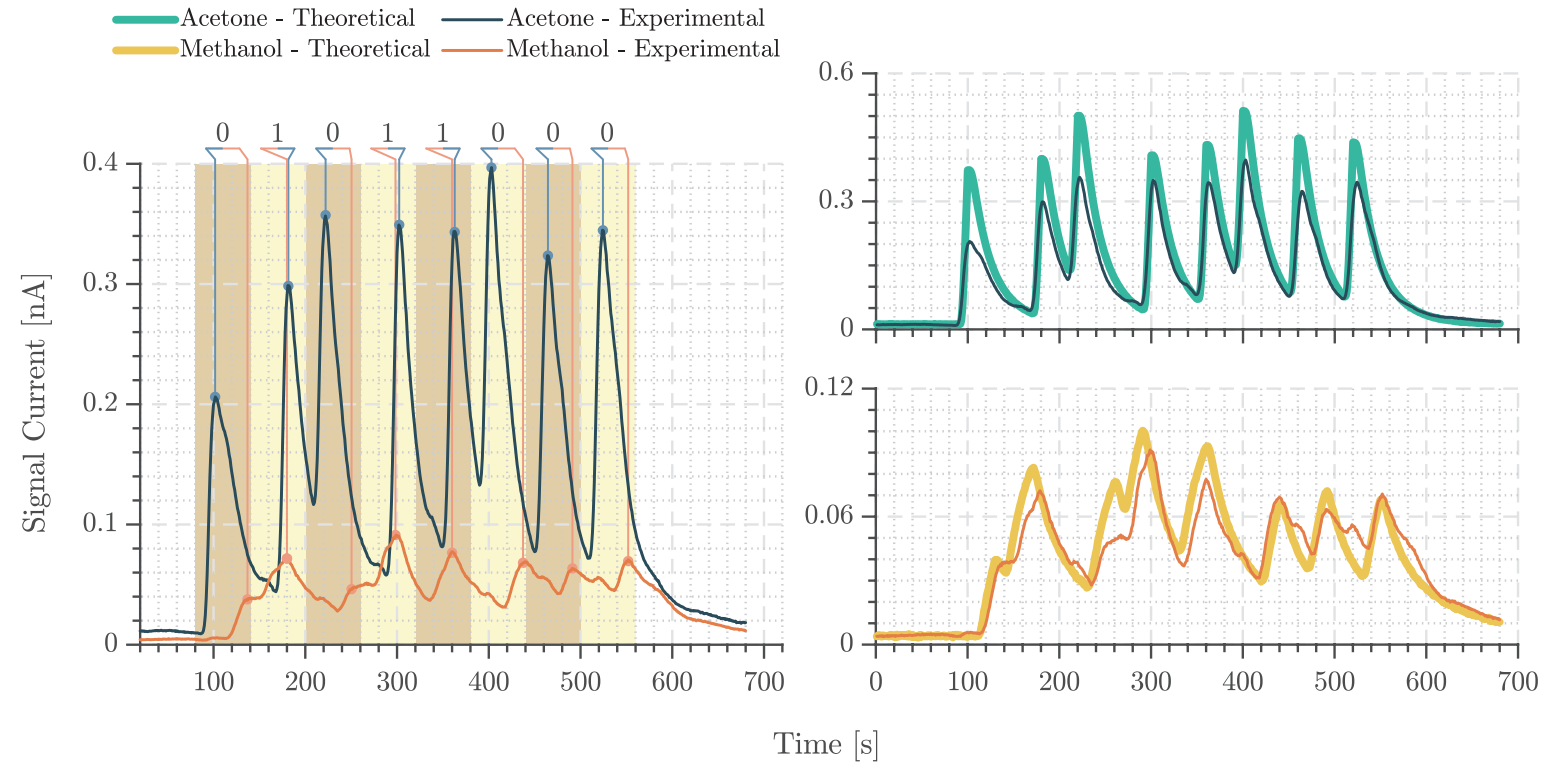

Fig. 11. Experimental transmission of acetone and methanol using ChToK

1) Experimental Analysis: This modulation, as can be seen has four parameters used to increase the number of bits transmitted. This, however opens up additional problems that are needed to be overcome, the primary one being the synchronization between the transmitter and the receiver. This can be mitigated by using one of the chemical channels as a clock, sending repeating pulses to keep the transmitter and receiver in-sync. This possible application can be seen in Figure 10 . In addition due to the chemicals' diffusivity, physical limits are imposed on the time unit $\delta T$ (i.e., Limit of Detection, Limit of Quantification, Sampling Rate).

An experimental transmission of ChToK was carried out with the parameters shown in Table IV The experimental results of the ChToK can be seen in Figure 11 The ASCII letter "X" (ASCII: 01111000) was transmitted by using acetone and methanol as signal chemicals, where in a given time frame of $T_{F}$, if the peak of the acetone appears first compared to methanol, it is given the symbol 0 , and symbol 1 is given for the inverse case.

From the experimental results, it can be observed that the signal of each chemical reach different peak amplitudes. This can be caused by various effects, such as inlet interactivity with chemicals, chemicals own interactivity or chemicals inherent properties. In each chemical period $T$, there are two distinguishable peaks, leading or lagging relative to acetone. The right side of Figure 11 is shown for the validation of the mathematical model in predicting the modulated signal for the transmission of acetone chemical. As can be seen the mathematical model, described in Section III], shows agreement $(\rho=0.96)$ with the experimentally transmitted signal.

\section{E. Chemical Ratio Modulation (ChRM)}

One evident method of increasing the carrying capacity of molecular communication is to employ multiple chemicals simultaneously. One such being the use of multiple chemical ratios to transmit information. Here information is encoded based on the ratio between two or more chemicals transmitting simultaneously. A diagram that represents the chemical ratio keying can be seen in Figure 12 .

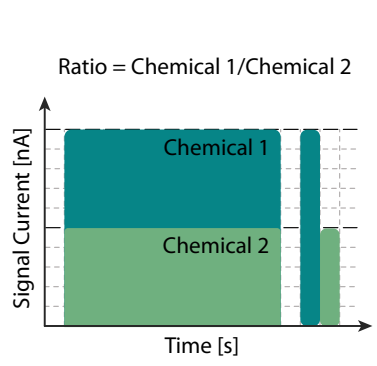

(a)

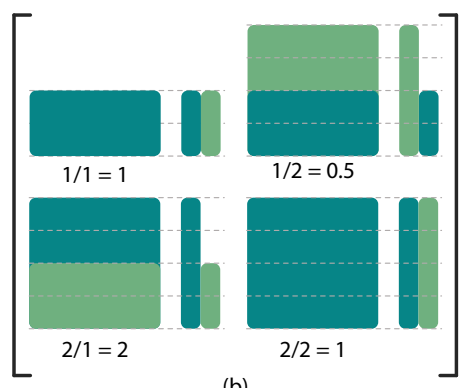

(b)
Fig. 12. (a) Diagram representing the chemical ratio keying (ChRM) (b) Possible combinations of $\operatorname{ChRM}(2,2)$.

1) Calculation of the modulation matrix: To mathematically define the modulation, two chemicals $\left(m_{A}, m_{B}\right)$ are used. Let $m_{A}$ and $m_{B}$ be the chemicals with the following set:

$$
\begin{array}{cc}
m_{A}=\left\{m_{A 0}, m_{A 1}, m_{A 2}, \ldots, m_{A i}\right\}, & m_{A} \subseteq \mathbb{N} \\
m_{B}=\left\{m_{B 0}, m_{B 1}, m_{B 2}, \ldots, m_{B j}\right\}, & m_{B} \subseteq \mathbb{N} .
\end{array}
$$

In this context the ratio is defined as the division of the chemicals:

$$
M(i, j)=\frac{m_{A i}}{m_{B j}}
$$

The matrix that gives the possible combinations of modulations is given as: 


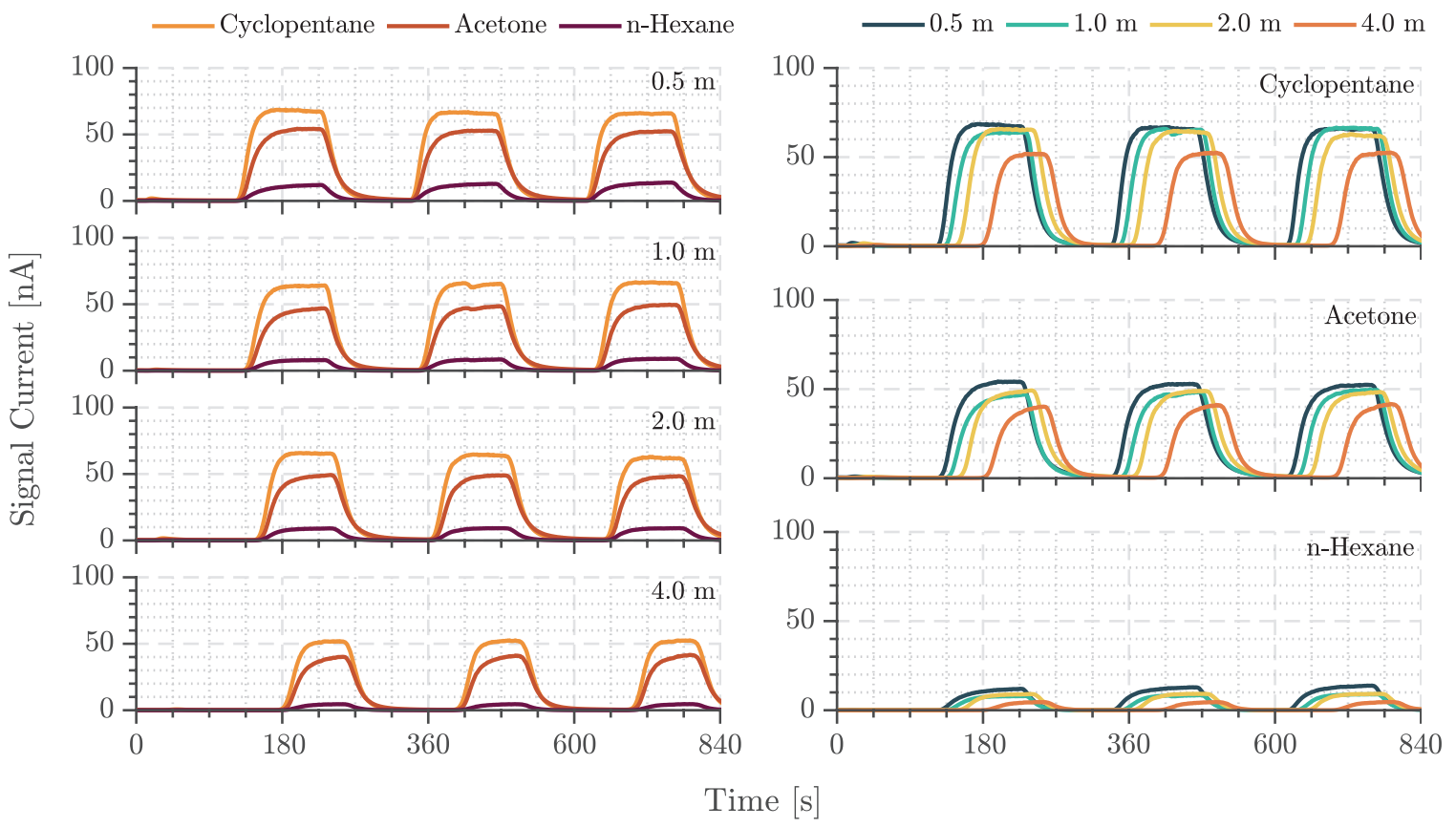

Fig. 13. Experimental transmission results of ChRM.

$$
M_{i, j}=\left(\begin{array}{cccc}
M(1,1) & M(1,2) & \ldots & M(1, j) \\
M(2,1) & M(2,2) & \ldots & M(2, j) \\
\vdots & \vdots & \ddots & \vdots \\
M(i, 1) & M(i, 2) & \ldots & M(i, j)
\end{array}\right)
$$

However, this matrix will possess values that are equal in terms of ratio (i.e., $4 / 2,6 / 3,8 / 4, \ldots$ ) which are not suitable for use in modulation and may cause confusion during decoding. The individuality of the ratios lies in co-prime numbers.

Two integers $m_{A}, m_{B}$ are co-prime if the only positive integer (factor) that divides both of them is 1. Any ratio between two co-primes will produce a unique value in which information can be encoded given enough precision. To calculate the number of generatable combinations, the RiemannZeta function is used with the following identity [50]:

$$
\zeta(s)=\sum_{n=1}^{\infty} \frac{1}{n^{s}} .
$$

The relation of the above function to the problem in hand is as follows. Let the sum definition of $\zeta(s)$ be the following expression:

$$
\zeta(s)=1+\frac{1}{2^{s}}+\frac{1}{3^{s}}+\frac{1}{4^{s}}+\frac{1}{5^{s}}+\cdots, \quad \mathcal{R}(s)>1 .
$$

From this series, to remove any number that is divisible by $2(2 \mid n)$ Eq. 230 is subtracted from 29p:

$$
\frac{1}{2^{s}} \zeta(s)=\frac{1}{2^{s}}+\frac{1}{4^{s}}+\frac{1}{6^{s}}+\frac{1}{8^{s}}+\frac{1}{10^{s}}+\cdots .
$$

The below equation represents the reciprocal of probability of $s$ amount of numbers not having a common divisor of 2 :
TABLE V

EXPERIMENTAL PARAMETERS FOR CHEMICAL RATIO KEYING (CHRK)

\begin{tabular}{rccc}
\hline Experimental Parameter & Symbol & Value & Unit \\
\hline Tracked ion & $\mathrm{m} / \mathrm{z}$ & $43^{1}, 42^{2}, 57^{3}$ & $\mathrm{Da}$ \\
Carrier flow & $Q$ & 1000 & $\mathrm{ml} / \mathrm{min}$ \\
Detection delay [34] & $t_{d}$ & $15^{1}, 14^{2}, 20^{3}$ & $\mathrm{~s}$ \\
Diffusivity in Air & $D$ & $0.124^{1}, 0.07^{2}, 0.11^{3}$ & $\mathrm{~cm}^{2} / \mathrm{s}$ \\
Inner Tube diameter & $\phi_{\text {in }}$ & 19.80 & $\mathrm{~mm}$ \\
Outer Tube diameter & $\phi_{\text {out }}$ & 24.25 & $\mathrm{~mm}$ \\
\hline
\end{tabular}

${ }^{1}$ acetone ${ }^{2}$ cyclopentane ${ }^{3}$ n-hexane

$$
\left(1-\frac{1}{2^{s}}\right) \zeta(s)=1+\frac{1}{3^{s}}+\frac{1}{5^{s}}+\frac{1}{7^{s}}+\frac{1}{9^{s}}+\cdots .
$$

This action is carried out to remove the common divisor of $3(3 \mid n)$ :

$$
\frac{1}{3^{s}}\left(1-\frac{1}{2^{s}}\right) \zeta(s)=\frac{1}{3^{s}}+\frac{1}{9^{s}}+\frac{1}{15^{s}}+\frac{1}{21^{s}}+\frac{1}{27^{s}}+\cdots .
$$

If this process is continued ad infinitum the following expression is obtained:

$$
\cdots\left(1-\frac{1}{5^{s}}\right)\left(1-\frac{1}{3^{s}}\right)\left(1-\frac{1}{2^{s}}\right) \zeta(s)=1 .
$$

The above expression gives the reciprocal probability of $s$ amount of numbers having no common divisors aside from 1 (i.e., co-prime):

$$
\zeta(s)=\sum_{n=1}^{\infty} \frac{1}{n^{s}}=\prod_{p \text { prime }} \frac{1}{1-p^{-s}} .
$$




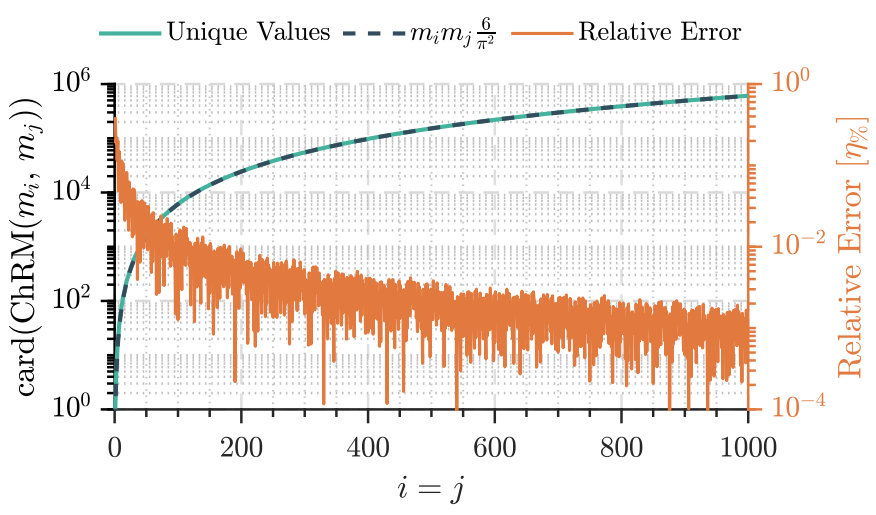

Fig. 14. Unique values generatable with ChRM.

The right-hand expression given in Eq. (34) is described as the Euler product which is an infinite product indexed by prime numbers. This connection allows the quantification and the calculation of the unique values of the modulation with high accuracy. While there are no closed form for odd values for Riemann-Zeta function, the closed form function for the even numbers can be expressed as:

$$
\zeta(2 n)=(-1)^{n+1} \frac{B_{2 n}(2 \pi)^{2 n}}{2(2 n) !} \quad n \in \mathbb{N},
$$

where $B_{2 n}$ is the Bernoulli number. Based on the aforementioned equations, the number of individual values in a modulation matrix used can be expressed as:

$$
\operatorname{card}\left(\operatorname{ChRM}\left(m_{1}, m_{2}, \cdots, m_{s}\right)\right)=\frac{1}{\zeta(s)} \prod_{i=1}^{i=s} m_{i}
$$

In the above-given example, two chemicals were used. The amount of modulated values are therefore can be approximated as:

$$
\phi_{A} \phi_{B} \frac{1}{\zeta(2)}=m_{A} m_{B} \frac{6}{\pi^{2}}
$$

In literature, the value of $\zeta(2)=\pi^{2} / 6$ is also known as the solution to the "Basel problem" [51]. Comparison of actual unique values to estimated ones along with estimation error can be seen in Figure 14 To test the proposed modulation scheme, an experiment was conducted with the parameters given in Table $\mathrm{V}$

2) Experimental Analysis: In this setup, a clear acrylic tube is used to initiate a long-distance transmission to show the resilience of the modulation to signal attenuation.

The experimental results can be seen collectively in Figure 13 From the Figure the effect of attenuation for three chemicals by increasing distance can be observed. Decrease of signal strength is to be expected. However, it must be noted that each chemical experiences different attenuation making some chemicals better at transmitting long distances than others.

As can be seen in Figure 13, the chemicals experience different attenuation rates. While cyclopentane and acetone experience similar attenuation, n-hexane experiences higher attenuation compared to the other two chemicals. This difference can be caused by the unique parameters of the chemical species used for sending the pulse. In addition each chemical achieves a different maximum amplitude which can also be caused by the ionization of samples, and their weight. The ratio of the two chemicals (cyclopentane/acetone) can be observed in Figure 15. Unlike the signal amplitude, the ratio value experiences less of a change, making it a better option to transmit information over long distances. However, the stability of the ratio value depends on the chosen chemical combinations. As can be seen from the Figure 15, some combinations can produce ratios that are maintained over distance whereas some ratios are not suitable for ratio modulation.

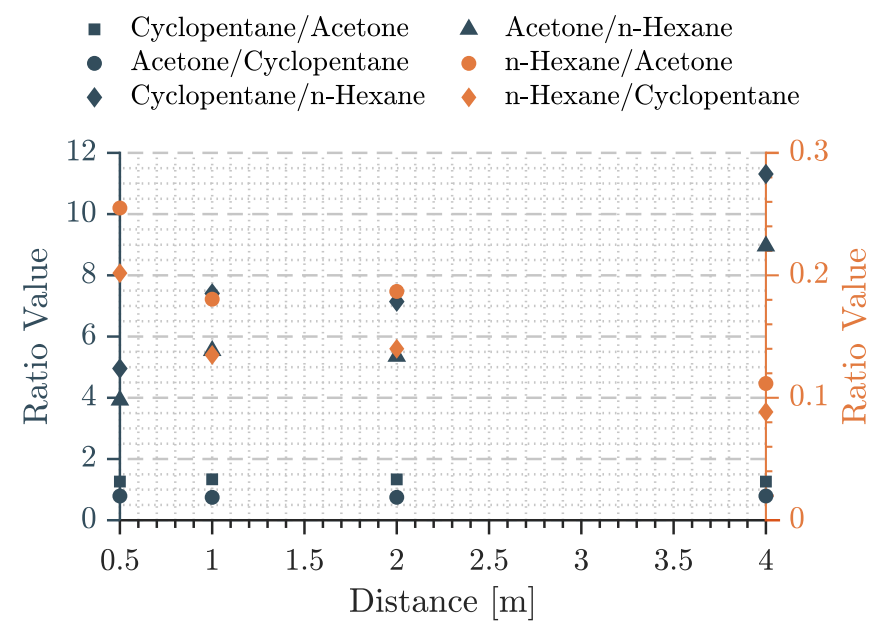

Fig. 15. Chemical ratio values from the ChRM experiment.

\section{CONClusion}

In this paper, experimental and analytical studies were conducted on the feasibility of transmitting multiple chemicals at the same time. In order to achieve this, an odor generator was used to generate and transmit chemicals and a quadrupole mass analyzer was utilized as a detector. The ability of the mass spectrometer, to distinguish and analyze multiple chemicals concurrently, makes them an invaluable tool for multiple chemical MC investigations. To mathematically validate the experiments and to understand the underlying behavior, advection-diffusion equation is used.

Experimentally, a multiple chemical transmission was carried out and shown to have strong agreement with mathematical model developed in this study. Experimentally the noise of the multiple chemical communication was analyzed and shown to exhibit additive white Gaussian noise behavior with distinct values for the mean and variance for each chemical. This characteristic presence gives rise to the notion that each chemical being transmitted with same signal strength will exhibit a different SNR value. The effect of chemical interference could also cause a decrease in SNR. This effect could be mitigated, however, if the chosen chemicals have no alignments in their mass spectrum. An example would be to 
use helium (He) and argon (Ar) as they have no matching peaks.

In this work, three modulation methods were proposed. The first one, ChQAM, based on concurrent transmission of multiple chemicals, is proposed and the SER is theoretically derived and verified by simulation described in Section III. Whereas the other two, ChToK and ChRM, are analyzed both theoretically and experimentally. ChToK is designed to send information through frames in which the position of the chemicals relative to each other in the frame defines the information content. This modulation method could also implement a clock inside the frame by designating a specific chemical as clock. This modulation method is also investigated by using acetone and methanol as information carriers. The experiment has shown the feasibility of implementing such modulation for possible applications. The third modulation method, ChRM, relies on the amplitude ratios of the transmitted signal. The cardinality of the modulation method is theoretically analyzed and a transmission using ChRM was experimented.

Three modulation methods proposed in the paper have different strengths and weaknesses depending on the environment. For example, it was shown that ChRM can transmit information over distance (up to $4 \mathrm{~m}$ ) with considerable degree of stability, However, this modulation has lower cardinality compared to other modulation methods proposed in this work. This shows that a modulation method that has a lower cardinality is not inherently inferior to one that has a higher value as they can be used in an application where the higher one cannot.

Future-work will focus on the exploration of novel modulation method that can be used in macro-scale molecular communications.

\section{ACKNOWLEDGMENT}

The research was funded from the Engineering and Physical Sciences Research Council (EPSRC) under the grant agreement: EP/M029425/1 'Creating a Stink - Investigating Olfactory Transport Streams'.

\section{REFERENCES}

[1] N. Farsad, H. B. Yilmaz, A. Eckford, C.-B. Chae, and W. Guo, "A Comprehensive Survey of Recent Advancements in Molecular Communication," IEEE Communications Surveys \& Tutorials, vol. 18, no. 3, pp. 1887-1919, 2016.

[2] W. Guo, T. Asyhari, N. Farsad, H. B. Yılmaz, B. Li, A. Eckford, and C.-B. Chae, "Molecular Communications: Channel Model and Physical Layer Techniques," IEEE Wireless Communications, vol. 23, no. 4, pp. 120-127, 2016.

[3] B. Atakan, Molecular Communications and Nanonetworks, $1^{\text {st }}$ ed. Springer-Verlag New York, 2014.

[4] F. Stajano, N. Hoult, I. Wassell, P. Bennett, C. Middleton, and K. Soga, "Smart Bridges, Smart Tunnels: Transforming Wireless Sensor Networks from Research Prototypes into Robust Engineering Infrastructure," Ad Hoc Networks, vol. 8, no. 8, pp. 872-888, 2010.

[5] S. Qiu, W. Guo, S. Wang, N. Farsad, and A. Eckford, "A Molecular Communication Link for Monitoring in Confined Environments," in International Conference on Communications Workshops (ICC). IEEE, 2014, pp. 718-723.

[6] A. E. Forooshani, S. Bashir, D. G. Michelson, and S. Noghanian, "A Survey of Wireless Communications and Propagation Modeling in Underground Mines," IEEE Communications Surveys \& Tutorials, vol. 15 , no. 4 , pp. 1524-1545, 2013.
[7] W. C. Agosta, Chemical Communication: the Language of Pheromones, $1^{\text {st }}$ ed. W. H. Freeman \& Co, 1992.

[8] R. K. Vander Meer, M. D. Breed, K. E. Espelie, and M. L. Winston, Pheromone Communication in Social Insects: Ants, Wasps, Bees and Termites, $1^{\text {st }}$ ed. Westview Press, 1997.

[9] J. Billen and E. D. Morgan, "Pheromone communication in social insects: sources and secretions," 1998.

[10] S. Kazadi, R. Goodman, D. Tsikata, D. Green, and H. Lin, "An autonomous water vapor plume tracking robot using passive resistive polymer sensors," Autonomous robots, vol. 9, no. 2, pp. 175-188, 2000.

[11] H. Ishida, T. Nakamoto, T. Moriizumi, T. Kikas, and J. Janata, "Plumetracking robots: A new application of chemical sensors," The Biological Bulletin, vol. 200, no. 2, pp. 222-226, 2001.

[12] A. Lilienthal, A. Zell, M. Wandel, and U. Weimar, "Sensing odour sources in indoor environments without a constant airflow by a mobile robot," in Robotics and Automation, 2001. Proceedings 2001 ICRA. IEEE International Conference on, vol. 4. IEEE, 2001, pp. 4005-4010.

[13] S. Larionova, N. Almeida, L. Marques, and A. T. de Almeida, "Olfactory coordinated area coverage," Autonomous Robots, vol. 20, no. 3, pp. 251260, 2006.

[14] H. A. Wheeler, "Fundamental limitations of small antennas," Proceedings of the IRE, vol. 35, no. 12, pp. 1479-1484, 1947.

[15] R. Bancroft, "Fundamental dimension limits of antennas," Centurion Wireless Technologies, pp. 1-14, 2002.

[16] J. S. McLean, "A re-examination of the fundamental limits on the radiation q of electrically small antennas," IEEE Transactions on antennas and propagation, vol. 44, no. 5, p. 672, 1996.

[17] I. Habibi, E. S. Emamian, and A. Abdi, "Quantitative analysis of intracellular communication and signaling errors in signaling networks," BMC systems biology, vol. 8, no. 1, p. 89, 2014.

[18] Y. Chahibi, "Molecular communication for drug delivery systems: A survey," Nano Communication Networks, vol. 11, pp. 90-102, 2017.

[19] T. Nakano, Y. Okaie, and J.-Q. Liu, "Channel model and capacity analysis of molecular communication with brownian motion," IEEE communications letters, vol. 16, no. 6, pp. 797-800, 2012.

[20] B. Atakan and O. B. Akan, "On channel capacity and error compensation in molecular communication," in Transactions on computational systems biology X. Springer, 2008, pp. 59-80.

[21] M. Pierobon and I. F. Akyildiz, "Capacity of a diffusion-based molecular communication system with channel memory and molecular noise," IEEE Transactions on Information Theory, vol. 59, no. 2, pp. 942-954, 2013.

[22] D. Arifler, "Capacity analysis of a diffusion-based short-range molecular nano-communication channel," Computer Networks, vol. 55, no. 6, pp. 1426-1434, 2011.

[23] F. Walsh, S. Balasubramaniam, D. Botvich, T. Suda, T. Nakano, S. F. Bush, and M. Ó. Foghlú, "Hybrid DNA and Enzyme based Computing for Address Encoding, Link Switching and Error Correction in Molecular Communication," in International Conference on Nano-Networks. Springer, 2008, pp. 28-38.

[24] M. S. Leeson and M. D. Higgins, "Forward error correction for molecular communications," Nano Communication Networks, vol. 3, no. 3, pp. 161-167, 2012.

[25] M. S. Leeson and M. D. Higgins, "Error Correction Coding for Molecular Communications," in IEEE International Conference on Communications (ICC). IEEE, 2012, pp. 6172-6176.

[26] L. Felicetti, M. Femminella, G. Reali, T. Nakano, and A. V. Vasilakos, "TCP-like Molecular Communications," IEEE Journal on Selected Areas in Communications, vol. 32, no. 12, pp. 2354-2367, 2014.

[27] V. Loscri, C. Marchal, N. Mitton, G. Fortino, and A. V. Vasilakos, "Security and Privacy in Molecular Communication and Networking: Opportunities and Challenges," IEEE Transactions on NanoBioscience, vol. 13, no. 3, pp. 198-207, 2014.

[28] N. Farsad, W. Guo, and A. W. Eckford, "Tabletop Molecular Communication: Text Messages Through Chemical Signals," PloS one, vol. 8 , no. 12, p. e82935, 2013.

[29] L. Grebenstein, J. Kirchner, R. S. Peixoto, W. Zimmermann, F. Irnstorfer, W. Wicke, A. Ahmadzadeh, V. Jamali, G. Fischer, R. Weigel, A. Burkovski, and R. Schober, "Biological Optical-to-Chemical Signal Conversion Interface: A Small-scale Modulator for Molecular Communications," IEEE Transactions on NanoBioscience, vol. 18, no. 1, pp 31-42, 2019.

[30] D. T. McGuiness, S. Giannoukos, A. Marshall, and S. Taylor, "Experimental Results on the Open-air Transmission of Macro-molecular Communication using Membrane Inlet Mass Spectrometry," IEEE Communications Letters, 2018. 
[31] D. T. McGuiness, S. Giannoukos, A. Marshall, and S. Taylor, "Parameter Analysis in Macro-scale Molecular Communications using AdvectionDiffusion," IEEE Access, vol. 6, pp. 46706-46717, 2018.

[32] D. McGuiness, A. Marshall, S. Taylor, and S. Giannoukos, "Asymmetrical inter-symbol interference in macro-scale molecular communications," in Proceedings of the 5th ACM International Conference on Nanoscale Computing and Communication. ACM, 2018, p. 13.

[33] B.-H. Koo, C. Lee, H. B. Yilmaz, N. Farsad, A. Eckford, and C.-B. Chae, "Molecular MIMO: from Theory to Prototype," IEEE Journal on Selected Areas in Communications, vol. 34, no. 3, pp. 600-614, 2016.

[34] S. Giannoukos, A. Marshall, S. Taylor, and J. Smith, "Molecular Communication over Gas Stream Channels using Portable Mass Spectrometry," Journal of the American Society for Mass Spectrometry, vol. 28, no. 11, pp. 2371-2383, 2017.

[35] E. De Hoffmann and V. Stroobant, Mass Spectrometry: Principles and Applications, 3rd ed. John Wiley \& Sons, 2007.

[36] M. Statheropoulos, G. Pallis, K. Mikedi, S. Giannoukos, A. Agapiou, A. Pappa, A. Cole, W. Vautz, and C. P. Thomas, "Dynamic Vapor Generator that Simulates Transient Odor Emissions of Victims Entrapped in the Voids of Collapsed Buildings," Analytical Chemistry, vol. 86, no. 8, pp. 3887-3894, 2014

[37] S. Giannoukos, B. Brkić, S. Taylor, and N. France, "Membrane Inlet Mass Spectrometry for Homeland Security and Forensic Applications," Journal of the American Society for Mass Spectrometry, vol. 26, no. 2, pp. 231-239, 2015.

[38] S. Giannoukos, B. Brkić, and S. Taylor, "Analysis of Chlorinated Hydrocarbons in Gas Phase using a Portable Membrane Inlet Mass Spectrometer," Analytical Methods, vol. 8, no. 36, pp. 6607-6615, 2016.

[39] S. Giannoukos, B. Brkić, S. Taylor, A. Marshall, and G. F. Verbeck, "Chemical Sniffing Instrumentation for Security Applications," Chemical Reviews, vol. 116, no. 14, pp. 8146-8172, 2016.

[40] S. Giannoukos, B. Brkić, S. Taylor, and N. France, "Monitoring of Human Chemical Signatures using Membrane Inlet Mass Spectrometry," Analytical Chemistry, vol. 86, no. 2, pp. 1106-1114, 2013.

[41] J. Crank, The mathematics of diffusion. Oxford university press, 1979.

[42] S. Wang, W. Guo, S. Qiu, and M. D. McDonnell, "Performance of Macro-scale Molecular Communications with Sensor Cleanse Time," in 21 st International Conference on Telecommunications (ICT). IEEE, 2014, pp. 363-368.

[43] W. W. Daniel et al., Applied Nonparametric Statistics, 1st ed. Houghton Mifflin, 1978

[44] S. Verdú et al., "A General Formula for Channel Capacity," IEEE Transactions on Information Theory, vol. 40, no. 4, pp. 1147-1157, 1994.

[45] T. M. Cover and J. A. Thomas, Elements of Information Theory, 1st ed John Wiley \& Sons, 2012.

[46] D. T. Mcguiness, S. Giannoukos, A. Marshall, and S. Taylor, "Modulation analysis in macro-molecular communications," IEEE Access, vol. 7, pp. $11049-11065,2019$.

[47] S. Kotz, N. Balakrishnan, and N. L. Johnson, Continuous Multivariate Distributions, Volume 1: Models and Applications, 2nd ed. John Wiley \& Sons, 2004

[48] C. E. Shannon, "A Mathematical Theory of Communication," Bell System Technical Journal, vol. 27, no. 3, pp. 379-423, 1948.

[49] H. D. Holland and K. K. Turekian, "Treatise on Geochemistry," 2004.

[50] E. C. Titchmarsh, E. C. T. Titchmarsh, D. Heath-Brown et al., The Theory of the Riemann Zeta-function, 1st ed. Oxford University Press, 1986.

[51] R. Ayoub, "Euler and the Zeta Function," The American Mathematical Monthly, vol. 81, no. 10, pp. 1067-1086, 1974. 